\title{
Tr1 cell-dependent active tolerance blunts the pathogenic effects of determinant spreading
}

\author{
Gizi Wildbaum, ${ }^{1}$ Nir Netzer, ${ }^{1}$ and Nathan Karin ${ }^{1,2}$ \\ ${ }^{1}$ Department of Immunology, and \\ ${ }^{2}$ Rappaport Family Institute for Research in the Medical Sciences, Bruce Rappaport Faculty of Medicine, \\ Technion, Haifa, Israel
}

\begin{abstract}
Experimental autoimmune encephalomyelitis (EAE) is a T cell-mediated autoimmune disease of the CNS. The current study shows that even in an acute episode of disease the autoimmune response spreads from one determinant on myelin basic protein (MBP) to the other linked determinant and that this spread plays a functional role in the pathogenesis of disease. The soluble form of each determinant could be used to induce Ag-specific T cell tolerance and reverse an ongoing disease. We show that the rapid effect of soluble peptide therapy is due to repolarization of autoimmune $T$ cells undergoing activation. We suggest that at least two different types of regulatory $\mathrm{T}$ cells participate in the induction of active tolerance. The first, yet to be fully characterized, functions in an IL-4-dependent manner. The second produces high levels of IL-10 and low levels of IL-4 (Tr1). We bring about completing evidence showing that these $\operatorname{Tr} 1$ cells play a pivotal role in the regulation of $T$ cell tolerance during determinant spread and that soluble peptide therapy with the determinant to which the autoimmune response spreads amplifies a de novo regulatory mechanism aimed to reduce the pathological consequences of determinant spreading.
\end{abstract}

J. Clin. Invest. 110:701-710 (2002). doi:10.1172/JCI200215176.

\section{Introduction}

Experimental autoimmune encephalomyelitis (EAE) is a T cell-mediated autoimmune disease of the CNS that serves as an animal model for human multiple sclerosis (MS) $(1,2)$. In EAE, and probably also MS, the autoimmune response of $\mathrm{T}$ cells to components of the CNS begins with recognition of a single or limited number of self-determinants. This response then expands into a reaction to several self-determinants on the same molecule, termed intramolecular determinant spreading, or to other molecules within the nervous system, known as intermolecular determinant spreading (3-8). Lewis rats, immunized with myelin basic protein (MBP) emulsified in CFA, first mount a primary $\mathrm{T}$ cell response to an encephalitogenic epitope encompassed by residues 68-86 (p68-86) of MBP. This response then spreads to a secondary epitope consisting of residues 87-99 (p87-99) (4, 5). These epitopes do not share cross-activating determinants. Yet, immunization of Lewis rats with each of these epitopes induces a response that

Received for publication February 1, 2002, and accepted in revised form July 9, 2002.

Address correspondence to: Nathan Karin, Rappaport Family Institute for Research in the Medical Sciences, Bruce Rappaport Faculty of Medicine, Technion, PO Box 9649, Haifa 31096, Israel. Phone: 972-4-8295232; Fax: 972-4-851-7797;

E-mail: nkarin@tx.technion.ac.il.

Conflict of interest: No conflict of interest has been declared.

Nonstandard abbreviations used: experimental autoimmune encephalomyelitis (EAE); regulatory T cells class 1 ( $\operatorname{Tr} 1)$; multiple sclerosis (MS); myelin basic protein (MBP); proteolipid apoprotein (PLP); Mycobacterium tuberculosis (MT); lymph node cell (LNC); effector line 1 (EL1); regulatory line 1 (RL1). spreads to the other determinant $(7,9)$. This type of "trans-acting" response has been observed in different models of MS (3, 6, 7, 9-12). Another encephalitogenic component of myelin is the proteolipid apoprotein (PLP). Lewis rats immunized with an encephalitogenic determinant of PLP (Ac224-240) develop a subpathogenic form of disease with an apparent histological, but not clinical, manifestation (13). During the course of EAE in the Lewis rat we were unable to observe transacting intermolecular spreading between each of the MBP determinants and PLPpAc224-240 (12).

Seven years ago we demonstrated, for the first time to our knowledge, that administration of the soluble form of the encephalitogenic determinant reverses an ongoing disease induced by $\mathrm{T}$ cells recognizing the same determinant (14). This process includes an intracellular elevation of $\mathrm{Ca}^{++}$at the target autoimmune cells (15). Neutralizing Ab's to IL-4 could reinstate disease susceptibility (16). This suggests that the tolerant state induced by soluble peptide therapy includes elicitation of regulatory $T$ cells. The rapid effect of soluble peptide therapy may also suggest that this therapy boosts a preexisting regulatory response. Hence, it is also not yet clear whether anti-IL-4 reverses the tolerant state because IL-4, by itself, acts as a regulatory cytokine or because IL-4 produced by these cells is essential for the function of other regulatory cells.

Several years ago Yu et al. demonstrated that administration of the soluble determinant to which the immune response spreads (spread determinant) could prevent a relapsing episode in SJL mice (17). Later, Vanderlugt et al. applied an alternative method of tolerance induction in the same experimental model to induce 
tolerance to the spread determinant of PLP (18). It is conceivable that regulatory $\mathrm{T}$ cells participate in the regulating of determinant spread, though this interesting subject has not yet been explored.

Antigen-specific $\mathrm{CD}^{+} \mathrm{T}$ cells fall into two major subsets; Th1 cells, which produces large amounts of IFN- $\gamma$ and TNF- $\alpha$ and low levels of IL-4, and Th2 cells, which mostly produce IL-4 and to a much lesser extent, IFN- $\gamma$ and TNF- $\alpha$ (19-29). Administration of neutralizing Ab's against IL-18, IL-12, or even the CXC chemokine IP-10 alters the Th1/Th2 balance toward high IL-4, low IFN- $\gamma$, and TNF- $\alpha-$ producing T cells, arrests leukocyte accumulation at the autoimmune site, and inhibits EAE (30-32). Hence, it is not fully understood whether alteration of the Th1/Th2 balance toward Th2 is beneficial due to the fact that these cells are regulatory cells, because their selection competes with Th1 selection, or because cytokines produced by these cells are essential for the function of other regulatory $\mathrm{T}$ cells. Other types of antigen-specific $\mathrm{CD} 4^{+} \mathrm{T}$ cells that were implicated in the regulation of the effector function of autoimmune $\mathrm{T}$ cells are the high TGF- $\beta$-producing T cells $(33,34)$, high IL-10, high IL-4, high IFN- $\gamma$ (35), or high IL-10, low IL-4-producing regulatory T cells class $1(\operatorname{Tr} 1)(36)$. $\mathrm{CD}^{+} \mathrm{CD} 25^{+}$immunoregulatory $\mathrm{T}$ cells have also become of central interest as potential regulators of tolerance in T cell-mediated autoimmunity (37-40).

The current study shows that soluble peptide therapy by the non-cross-activating determinant to which the immune response spreads, polarizes in vivo $\operatorname{Tr} 1$ cells. These cells produce high levels of IL-10 in response to the determinant to which the immune response spreads, but not in response to the determinant with which the disease was induced. The ability of such cells to suppress EAE and the competence of anti-IL-10 Ab to reverse tolerance induced by soluble peptide therapy with this determinant further support their potential regulatory role.

\section{Methods}

Rats. Female Lewis rats, approximately 6 weeks old were purchased from Harlan Biotech Israel Ltd. (Jerusalem, Israel) and maintained under special pathogen free conditions in our animal facility.

Peptide antigens. MBPp87-99, VHFFKNIVTPRTP, MBPp68-86, YGSLPQKSQRSQDENPV, P2p57-83 (41), and PLPpAc224-240, Ac-L S I C K T A E F Q M T F H L F I-NH were all synthesized on a MilliGen 9050 peptide synthesizer by standard 9-fluorenylmethoxycarbonyl chemistry. Peptides were purified by HPLC. Structure was confirmed by amino acid analysis and mass spectroscopy. Only peptides that were greater than $95 \%$ pure were used in our study.

Active induction of EAE or experimental autoimmune neuritis. MBPp87-99 or P2p57-83 at a concentration of 1 $\mathrm{mg} / \mathrm{ml}$ were dissolved in PBS and emulsified with an equal volume of incomplete Freund's adjuvant supplemented with $4 \mathrm{mg} / \mathrm{ml}$ heat-killed Mycobacterium tuberculosis (MT) H37Ra in oil (Difco Laboratories, Detroit,
Michigan, USA). Rats were immunized subcutaneously in the hind footpads with $0.1 \mathrm{ml}$ of the emulsion and monitored daily for clinical signs by an observer blind to the treatment protocol. EAE was scored as follows: 0 , clinically normal; 1 , flaccid tail; 2 , hind limb paralysis; 3 , front and hind limb paralysis; 4 , total body paralysis. Experimental autoimmune neuritis (EAN) was scored as follows: 0 , clinically normal; 1 , flaccid tail; 2 , hind limb paralysis.

$T$ cell lines and clones. MBPp68-86 T cell lines were selected as done previously (42). In all cultures, stimulation medium was enriched with $100 \mathrm{U} / \mathrm{ml}$ recombinant rat IL-10 (522-RL; R\&D Systems Inc., Minneapolis, Minnesota, USA). Clones were selected after two selection cycles of the line by limiting dilution technique (one cell per three wells) according to the basic protocol described by Ben-Nun et al. (43). All clones were continually supplemented with $10 \%$ Con A supernatant as a source of IL-2.

To ensure the purity of clones W- 11 and W- 2 that were obtained by limited dilution, each clone was subjected to RT-PCR with consensus primers (44), followed by sequencing of the VDJ region.

Antigen-specific T cell proliferation assays. Lewis rats were immunized with MBPp87-99/CFA as described above. Thirteen days later spleen cells were suspended in stimulation medium and cultured in U-shaped 96-well microculture plates $\left(2 \times 10^{5}\right.$ cells/well) for 72 hours in the presence of $100 \mu \mathrm{M}$ peptide antigen at $37^{\circ} \mathrm{C}$ in humidified air containing 7.5\% $\mathrm{CO}_{2}$. Each well was pulsed with $2 \mu \mathrm{Ci}$ of $\left[{ }^{3} \mathrm{H}\right]$-thymidine (specific activity $10 \mathrm{Ci} / \mathrm{mmol}$ ) for the final 16 hours. The cultures were then harvested on fiberglass filters. Results are shown as mean cpm of triplicates \pm SE.

$A b$ 's. Affinity-purified neutralizing $\mathrm{Ab}$ against IL-4 (anti-rat IL-4; 500-P94) and IL-10 (anti-rat IL-10; 500-P139) were acquired from PeproTech Inc.(Rocky Hill, New Jersey, USA) and used to neutralize IL-4 and IL-10 in all our in vivo and in vitro experiments.

Cytokine determination in primary cultures. Spleen cells or lymph node cells (LNCs) from EAE donors were stimulated in vitro $\left(10^{7}\right.$ cells $\left./ \mathrm{ml}\right)$ in 24-well plates (Nalge Nunc International, Naperville, Illinois, USA) with 100 $\mu \mathrm{M}$ peptide antigen. After 72 hours of stimulation, supernatants were assayed for the protein level of various cytokines using the commercially available ELISA kits we have specified elsewhere $(31,45)$. Additional semi-kits that were used in the current study were IL-10 (first Ab: 240610, standard catalog 25071V; second Ab: 24072D; PharMingen, San Diego, California, USA) and TGF- $\beta$ (first Ab: MAB240, standard catalog 240B-002; second Ab: 2BAF240; R\&D Systems Inc.).

FACS analysis. FACS analysis was conducted according to the basic protocol we used previously (46). Mouse anti-rat CD4:biotin (MCA55B; Serotec Ltd., Oxford, United Kingdom) was used for gating of $\mathrm{CD}^{+}$ T cells. Spectral red conjugate (MCA55B; Southern Biotechnology Associates, Birmingham, Alabama, USA) was then used as a second step. Intracellular 


\section{Table 1}

Determinant spread of the immune response during MBPp87-99/ CFA-induced EAE
$\mathrm{Ag}$

MBPp87-99

MBPp68-86

PLPpAc224-240
Proliferation

Day 11 (onset)

$6.2 \pm 0.4$

$5.3 \pm 0.8$

$1.2 \pm 0.2$
(Stimulation index $\pm \mathrm{SE}$ )

Day 14 (peak of disease)

$4.7 \pm 0.8$

$9.6 \pm 1.2$

$1.4 \pm 0.2$
Lewis rats were immunized with MBPp87-99/CFA to induce active EAE. At the onset of disease (day 11) and when animals reached maximal clinical score (day 14) cervical LNCs were determined for the development of proliferative response to MBPp87-99, MBPp68-86, and PLPpAc224-240. Results are shown as mean stimulation index of three samples \pm SE. Background counts (without addition of antigens) were 1,350 $\pm 80 \mathrm{cpm}$ (day 11) and 1,780 \pm 140 cpm (day 14).

staining of IL-4 and IL-10 was done using a commercially available kit (LEUCOPERM, BUF9; Serotec Ltd.) according to the manufacturer's protocol. FITClabeled mouse anti-rat IL-10 mAb (ARC9108; Biosource Europe SA, Nivelles, Belgium) and phycoerythrin-labeled mouse anti-rat IL-4 mAb (24055A; Biosource Europe SA) were used for direct staining. Brefeldin A was added to cells for the last 6 hours as described previously by Openshaw et al. (47). Cells were analyzed using a FACSCalibur (Becton Dickinson Immunocytometry Systems, Mountain View, California, USA). Data were collected for 10,000 events and analyzed using a Cell Quest software program (Becton Dickinson Immunocytometry Systems).

Histopathology. Frozen sections of the same area of the lumbar region of the spinal cord were stained with hematoxylin and eosin. Each section was evaluated without knowledge of the treatment status of the animal. The following scale was used: 0 , no perivascular lesions; 1 , one to five perivascular lesions per section with minimal parenchymal infiltration; 2 , five to ten perivascular lesions per section with parenchymal infiltration; and 3, more than ten perivascular lesions per section with extensive parenchymal infiltration. The mean histological score \pm SE was calculated for each treatment group. (Representative photomicrographs are shown in Figure 2.)

Statistical analysis. Significance of differences was examined using Student $t$ test. $P$ values less than 0.05 were considered significant. Mann-Whitney sum of ranks test was used to evaluate significance of differ- ences in mean of maximal clinical score. $P$ values less than 0.05 were considered significant.

\section{Results}

Lewis rats were immunized with MBPp87-99/CFA to induce active EAE and followed for the development of $\mathrm{T}$ cell proliferative response to MBPp87-99, MBPp68-86, or PLPpAc224-240 (Table 1). At the onset of disease, these rats mounted an apparent response to both MBP determinants (stimulation index $=6.2 \pm 0.4$ and $5.3 \pm 0.8$, respectively), but not to PLPpAc224-240 (stimulation index $=1.2 \pm 0.2$ ) or to the neurotogenic peptide of P2 (P2p57-81, not shown). At the peak of disease (day 14) the response against the determinant to which the response spread (MBPp68-86) was even significantly higher than the one developed against the determinant with which disease was induced (Table $1 ; 9.6 \pm 1.2$ vs. $4.7 \pm 0.8$, $P<0.001$ ). Effector line 1 (EL1; see Table 2 and Figure 5) was selected in response to $\mathrm{p} 68-87$ from rats that were immunized only with p87-99/CFA. Even after a single selection cycle (second stimulation) the pre-line was found to be mono specific (i.e., proliferates in response to p68-86 and not p87-99) and encephalitogenic $\left(2 \times 10^{6}\right.$ cells transferred EAE to six of six rats). This line clearly shows that even the acute model of MS in the Lewis rat includes determinant spread of pathogenic T cells. At no time we could identify determinant spread of the autoimmune response to PLPpAc224-240. Administration of soluble MBPp87-99 or MBPp68-86, but not PLPpAc224-240 or P2p57-81, led to a very fast remission from disease induced by MBPp87-99/CFA (Figure 1, $P<0.001$ ). This further substantiates the previous observations of Yu et al. in the SJL model of disease (17). Neutralizing Ab's to IL-4, but not to IL-10, could totally abrogate the tolerant state acquired by administration of soluble MBPp87-99 to rats with developing MBPp87-99/CFA disease (Figure 1b). In contrast, neutralizing Ab's to IL-10, but not to IL-4, could break down tolerance induced by the spread determinant (Figure 1c). Neither anti-IL-4 nor anti-IL-10 Ab's $(100 \mu \mathrm{g} / \mathrm{rat})$ could significantly affect the course of disease in control EAE rats (Figure 1d). Thus, anti-IL-10 Ab reverses tolerance induced by a determinant to which the autoimmune response spreads. Normal rabbit IgG (100 $\mu \mathrm{g} / \mathrm{rat}$ ) from preimmunized rabbits had no effect on

\section{Table 2}

Cytokine production by MBPp68-86-specific encephalitogenic and regulatory T cells

\begin{tabular}{|c|c|c|c|c|c|}
\hline \multirow[t]{2}{*}{ Line/clone } & \multirow[t]{2}{*}{ Biological function } & \multicolumn{4}{|c|}{ Cytokine production } \\
\hline & & $\mathrm{IFN}-\gamma(\mathrm{ng} / \mathrm{ml})$ & IL-4 (pg/ml) & $\mathrm{IL}-10(\mathrm{pg} / \mathrm{ml})$ & TGF- $\beta(\mathrm{pg} / \mathrm{ml})$ \\
\hline Line EL-1 & Encephalitogenic & $28.8 \pm 4$ & $105 \pm 24$ & $280 \pm 60$ & ND \\
\hline Line RL-1 & Regulatory & $14.7 \pm 1.2$ & $145 \pm 20$ & $1,850 \pm 230$ & ND \\
\hline Clone W-2 & Encephalitogenic & $26.5 \pm 7$ & $95 \pm 18$ & $320 \pm 45$ & $210 \pm 32$ \\
\hline Clone W-11 & Regulatory & $8.2 \pm 0.4$ & $130 \pm 14$ & $2,200 \pm 140$ & $230 \pm 25$ \\
\hline
\end{tabular}

Line EL-1, line RL-1, clone W-2, and clone W-11 were cultured with $100 \mu \mathrm{g} / \mathrm{ml}$ p68-86 for $48-72$ hours. Cytokine levels were then determined by ELISA and are presented as mean of triplicates \pm SE. ND, not detectible. 


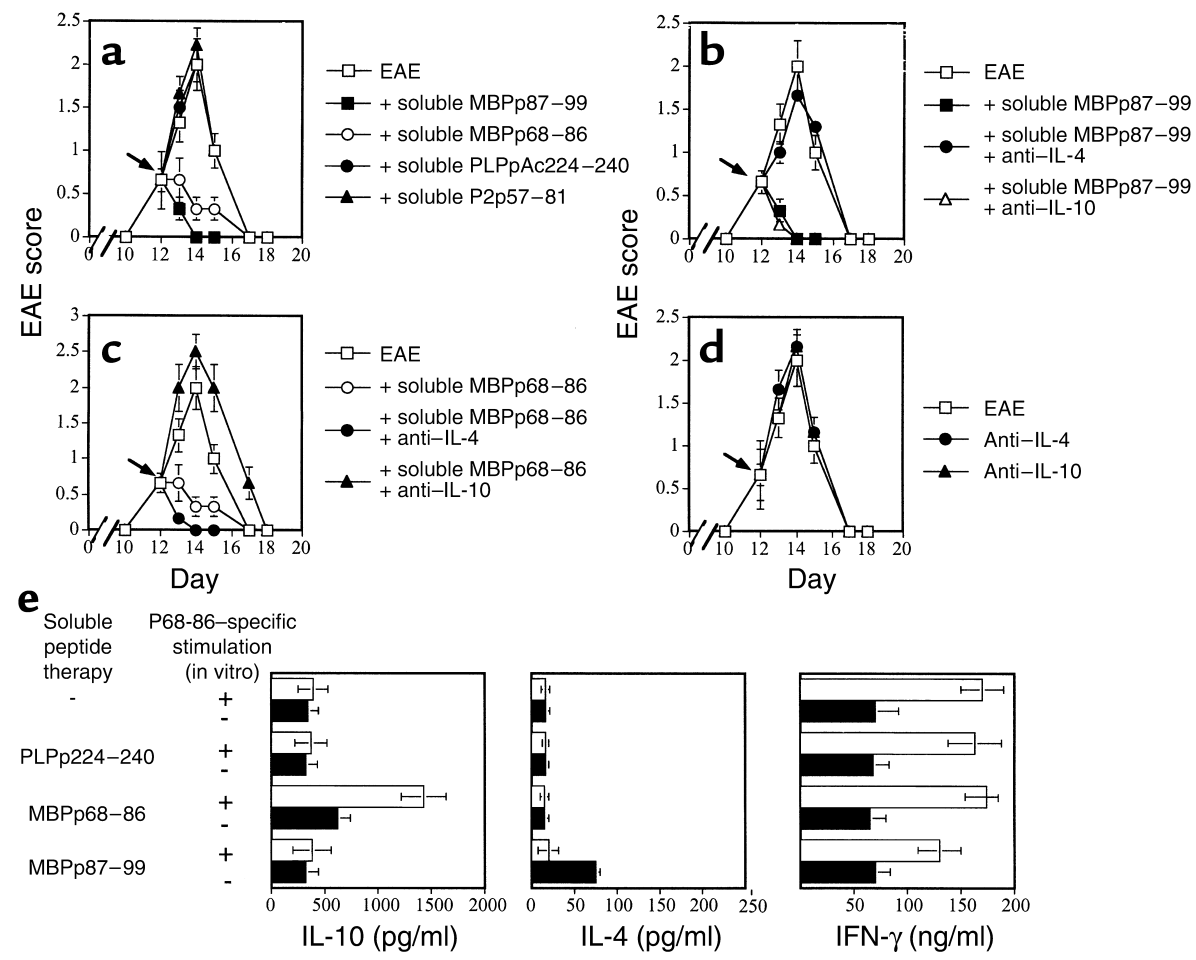

\section{Figure 1}

(a-d) Groups of nine Lewis rats were immunized with MBPp87-99/CFA to induce active EAE. Just after the onset of disease (day 11) these rats were subjected to soluble peptide administration of MBPp87-99, MBPp68-86, PLPpAc224-240, or P2 (P2p57-81). Repeated administrations were given in a 12-hour interval during the subsequent 24 hours, and monitoring was done for clinical signs of disease (a). On day 11 following the first peptide administration, some of the groups that were subjected to soluble peptide therapy with MBPp87-99 (b) or MBPp68-86 (c) were also administered with neutralizing Ab's to IL-4 or IL-10. (d) The kinetics of disease in rats that were administered with each of the above Ab's without soluble peptide therapy. Mean EAE score \pm SE of six rats per group is shown. Either normal rabbit IgG or affinity-purified polyclonal Ab's from preimmunized rabbits were used as control Ab's to anti-IL-4 and anti-IL-10. Their administration had no effect on the manifestation of disease, or on the suppressive effect of either soluble MBPp68-86 or MBPp87-99. (e) Levels of IL-10, $\mathrm{IL}-4$, and IFN- $\gamma$ obtained during the response to the spread determinant in spleen cells of rats that were subjected to active EAE induced by p87-99/CFA and subjected to soluble peptide therapy with various epitopes as described above. Spleen cells were obtained 24 hours after soluble peptide therapy was administered. Results are shown as mean of triplicates \pm SE.

the manifestation of disease, nor on the suppressive effect of either soluble MBPp68-86 or MBPp87-99 (data not shown).

On day 13, just before the peak of disease in control rats, spinal cord samples from these rats were histologically analyzed. Figure 2 shows representative sections of 12 sections from three rats per group. The repeated administration of MBPp68-86 to rats with developing EAE induced by MBPp87-99/CFA led to a dramatic decrease of perivascular infiltrating mononuclear cells (Figure 2, b vs. c, mean histological score of $2.5 \pm 0.16$ vs. $0.83 \pm 0.12, P<0.001$ ), which could well explain the marked reduction in clinical scores in these animals (Figure 1a). Anti-IL-10 Ab's that abrogated this effect (Figure 1c) also reinstated the accumulation of perivascular infiltrating mononuclear cells (Figure 2d, $2 \pm 0.33$, $P<0.001$, compared with Figure 2c).

Various types of cells could secrete IL-10. We have identified the cells that produce high levels of IL-10 following soluble peptide therapy. In this experiment, rats were administered with p87-99/CFA to induce active EAE and then subjected to soluble peptide ther- apy with MBPp87-99, MBPp68-86, or PLPp224-240. Spleen $T$ cells from each of these groups were then cultured in the presence or absence of the tolerizing determinant (p68-86) and tested for cytokine production (Figure 1e). An elevated production of IL-10 $(P<0.01)$ could be identified only in primary cultures from EAE rats that were treated with the spread determinant (p68-86). In vitro addition of this determinant led to a remarkable acceleration $(P<0.0001)$ in the production of this cytokine (Figure 1e). Interestingly, primary $T$ cells isolated from rats that were subjected to soluble peptide therapy with the spread determinant and that exhibited a markedly reduced response to this determinant (which could provide a partial explanation to the in vivo tolerant state) continued to produce (ex vivo) high levels of IFN- $\gamma$ (Figure 1e), a cytokine with a pleiotropic function in the regulation of $\mathrm{T}$ cell-mediated autoimmunity.

The rapid effect of soluble peptide therapy may suggest that it amplifies a preexisting response. In an attempt to follow the kinetics of the accumulation of high IL-10-producing $\mathrm{T}$ cells in lymph nodes that 
drain the autoimmune site (cervical LNCs) Lewis rats were injected with MBPp87-99/CFA to induce active $\mathrm{EAE}$, and at the onset of disease were subjected to soluble peptide therapy with p87-99 or p68-86. Twentyfour hours later cervical LNCs (Figure 3, a-d), popliteal LNCs (not shown), and spleen cells (Figure $3, \mathrm{e}-\mathrm{f}$ ) were subjected to flow-cytometry analysis of IL-4 versus IL- 10 production by $\mathrm{CD} 4^{+} \mathrm{T}$ cells. The data presented in Figure 3 represent one of three independent experiments with very similar results. Figure $3, b$ and $d$, show the appearance of numerous $\operatorname{Tr} 1$ cells in cervical lymph nodes (LNs) of control EAE rats that were not subjected to soluble peptide therapy (Figure $3 \mathrm{~d}, 1.2 \%)$ or were treated with the determinant with which disease was induced (Figure 3b, 1.6\%). This population markedly increased in cervical LNs from rats treated with the determinant to which the response spread (Figure 3c, 6.2\%). Soluble peptide therapy with the epitope of spread also significantly induced $\operatorname{Tr} 1$ cells in the spleen (Figure 3, g vs. e or h, $3.7 \%$ vs. $0.3 \%$ and $0.9 \%, P<0.001$ ). The differences in the accumulation of these cells in cervical LNs and spleen (Figure 3, c vs. g, $6.5 \%$ vs. $3.7 \%$ ) could be explained, in part, by the nature of these lymph nodes that drain the autoimmune site. These results are striking because LNCs and spleen cells from tolerized animals were not subjected to Ag-specific in vitro activation. They clearly demonstrate a rapid and massive appearance of $\operatorname{Tr} 1$ cells in animals that were subjected to soluble peptide therapy with the spread determinant.

To determine the specificity and potential function of these cells, cervical LNCs were isolated from rats that were injected with p87-99/CFA to induce active EAE and then treated with soluble p68-86 to suppress the disease (see Figure 1 and Figure 3). These cells were determined for their ability to mount epitope-specific proliferative responses to the spread determinant (p68-86), the determinant with which disease was induced (p87-99), the whole MBP including p87-99 and p68-86, and a control Ag (MT). The results shown in Figure 4 represent data obtained in four different experiments with very similar observations. Even though each of these responses was significantly reduced in tolerized animals $(P<0.01)$ suppression of the response in the presence of the tolerizing determinant (MBPp68-86), alone or as a part of the whole MBP, was more significant than in the absence of this determinant (Figure 4a, $P<0.001)$ ). Anti-IL-10 (Figure $4 c$ ), but not anti-IL-4 (Figure $4 \mathrm{~b}$ ) Ab could then restore the suppressed response. This clearly shows that the regulatory cells, once activated in vivo, can suppress Ag-specific responses in a bystander manner, yet the presence of the tolerizing determinant further increases their suppressive competence.

The effect of soluble peptide therapy with the direct epitope with which the disease was induced was also recorded in this experiment. The addition of MBPp87-99 to primary cultures from rats that were treated with MBPp87-99 significantly increased IL-4 production $(75 \pm 6 \mathrm{pg} / \mathrm{ml}$ vs. $24 \pm 4 \mathrm{pg} / \mathrm{ml}, P<0.01)$, but had no effect on the level of IL-10 produced by these cells $(460 \pm 40 \mathrm{pg} / \mathrm{ml}$ vs. $410 \pm 55 \mathrm{pg} / \mathrm{ml})$. This further supports the hypothesis that different regulatory $\mathrm{T}$ cells direct the regulation of the immune response during determinant spread than those induced by soluble therapy with the determinant with which disease was induced. Surprisingly, neither ant-IL-10 Ab nor anti-IL-4 Ab could abrogate suppression induced following soluble peptide therapy with the determinant with which disease was established (data not shown). This also suggests that IL-4 is not a suppressor cytokine, but is probably important for the in vivo maintenance/function of regulatory cells induced by direct soluble peptide therapy.
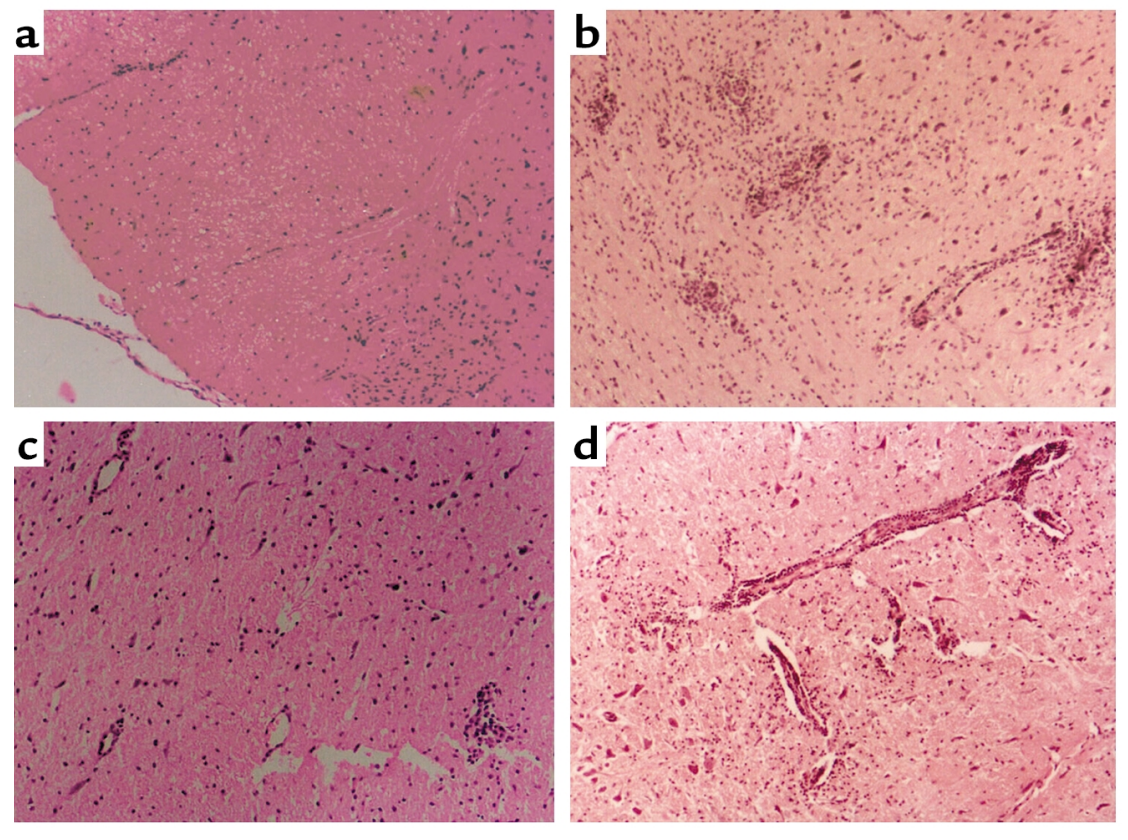

\begin{abstract}
Figure 2
(a-d) Thirteen days after immunization with MBPp87-99/CFA to induce active $E A E$, the lower lumbar region of the spinal cord of EAE rats that were not subjected to soluble peptide therapy (b), subjected to therapy with soluble MBPp68-86 (c), or subjected to therapy with soluble MBPp68-86, and then with neutralizing Ab's to IL-10 (d), were evaluated for histological scores of EAE in comparison with spinal cord sections from naive Lewis rats of comparable age to the experimental rats (a). Each section (except for control naive rats) represents 12 sections from three rats that were analyzed by an observer blind to experimental procedure. The full analysis of these samples is specified in the text. Hematoxylin and eosin-stained sections are shown at $\times 10$.
\end{abstract}



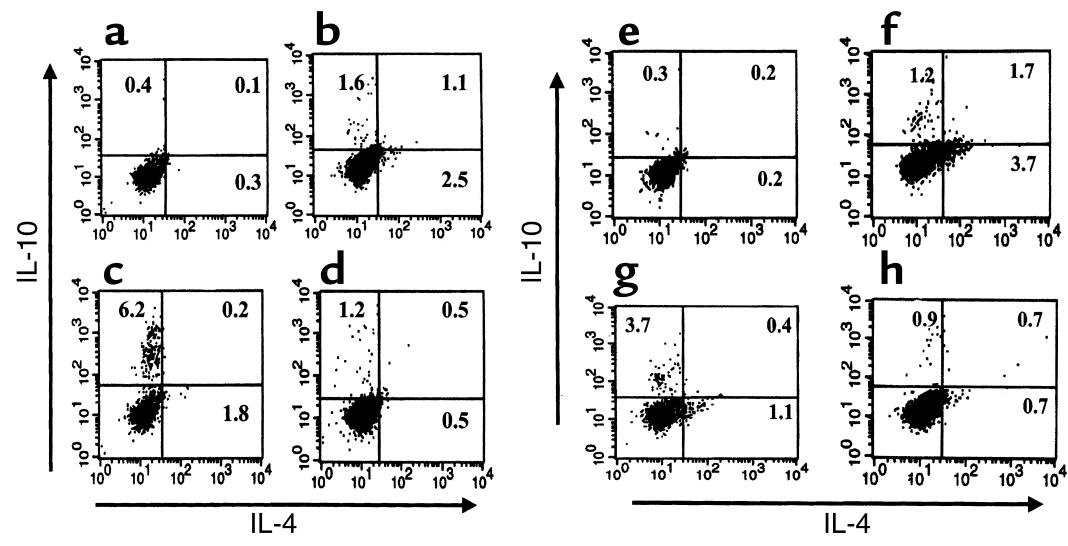

Figure 3

Lewis rats were subjected to active EAE induced by MBPp87-99/CFA and at the onset of disease were subjected to soluble peptide therapy with p87-99 or p68-86. Twenty-four hours later, cervical LNCs (a-d) and spleen (e-h) were subjected to flow cytometry analysis of IL-4 vs. IL-10 production by CD4 ${ }^{+} \mathrm{T}$ cells, gated by anti-CD4 ${ }^{+} \mathrm{Ab}$ as described in Methods. ( $\mathbf{a}$ and $\mathbf{e}$ ) Analysis of cells from a naive rat. (b and $\mathbf{f}$ ) Analysis of cells from EAE rats treated with the soluble form of the determinant with which disease was induced (MBPp87-99). (c and $\mathbf{g}$ ) Analysis of cells from EAE rats treated with the soluble form of the determinant of spread (MBPp68-86). (d and $\mathbf{h}$ ) Analysis of cells from EAE rats treated with PBS.

that were, or were not, subjected to soluble peptide therapy with the spread determinant (p68-86). After four selection cycles, each line included more than $98 \% \mathrm{CD}^{+} \mathrm{T}$ cells (W3/25 positive). The control line (EL1) produced very low levels of IL-10 and IL-4 (Table 2, $280 \pm 60$ $\mathrm{pg} / \mathrm{ml}$ and $105 \pm 24 \mathrm{pg} / \mathrm{ml}$, respectively, in response to $100 \mu \mathrm{g} / \mathrm{ml}$ MBPp68-86). When administered to naive Lewis rats $\left(5 \times 10^{6}\right.$ cells per rat) EL1 could effectively transfer disease (incidence six of six, mean maximal score $2 \pm 0$ ). When transferred to rats with developing EAE, these effector $T$ cells aggravated the severity of disease (Figure 5, a and b, $P<0.05$ ). In contrast, MBPp68-86-specific T cells selected from rats treated with soluble MBPp68-86 (which we named regulatory line $1, \mathrm{RL} 1$ ), produced high levels of IL-10 (Table 1, $1,850 \pm 230 \mathrm{pg} / \mathrm{ml} P<0.0001$ com-

To verify that the heterologous peptide induces IL-10 because it triggers a mechanism designed to prevent determinant spreading and not because it is simply related to the nature of the peptide antigen, we have repeated the same experimental protocol, but Lewis rats were administered MBP68-86/CFA to develop active $\mathrm{EAE}$, and 1 day after the onset of disease, when an apparent response to MBPp87-99 could also be recorded in cervical LNs, these rats were repeatedly administered with soluble p87-99. The proliferative response developed in these animals to the spread determinant was markedly suppressed, but could be restored by anti-IL-10, and not anti-IL-4 Ab (data not shown). This generalizes our basic findings that heterologous peptide induces IL-10 production during determinant spread.

Thereafter, MBPp68-86-specific T cell lines were selected under the same conditions (i.e., stimulation medium enriched with IL-10 and IL-2) from EAE rats pared with EL1), but not IL-4 $(145 \pm 20 \mathrm{pg} / \mathrm{ml})$. These cells were nonencephalitogenic (at a dose of $10 \times 10^{6}$ cells per rat incidence 0 of 12 ), and when administered to EAE rats (at a dose of $2 \times 10^{6}$ cells per rat) they could induce a high state of EAE resistance (Figure 5, a and b, $P<0.001)$. Their administration to rats with developing active EAN had no effect on the development progression of the disease (Figure $5 \mathrm{c}$ ). Thus, the protective effect of these $T$ cells is disease specific. Consistent with the observations presented in Figure 1, anti-IL-10, but not anti-IL-4 or control rabbit IgG, could entirely reverse the protective effect of our high IL-10-producing RL1 (not shown).

We have then selected $12 \mathrm{CD}^{+} \mathrm{T}$ cell clones from RL1. Eleven of them produced high levels of IL-10, and one (clone W-2) has been defined as a Th1 type, producing low levels of IL-10 and IL-4, high levels of IFN- $\gamma$, and low levels of TGF- $\beta$ (Table $1,320 \pm 45$

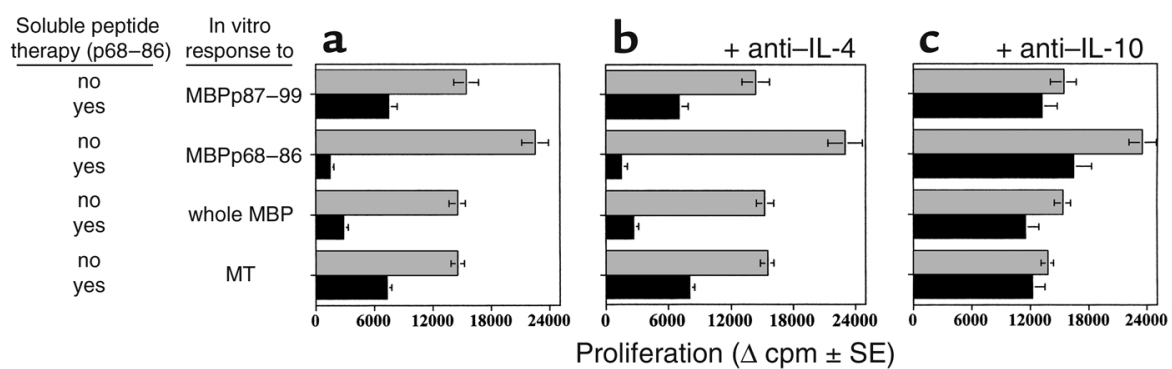

Figure 4

Thirteen days after immunization with MBPp87-99/CFA to induce active EAE, cervical lymph node cells and spleen cells (not shown) of EAE rats were determined for their ability to mount a proliferative response to MBPp87-88, MBPp68-86, whole Guinea-pig MBP, and MT. (a) The proliferative responses to each antigen. (b) The proliferative responses to each antigen in the presence of rabbit anti-rat neutralizing Ab's $(10 \mu \mathrm{g} / \mathrm{ml})$ to IL-4. (c) The proliferative responses to each antigen in the presence of rabbit anti-rat neutralizing Ab's $(10 \mu \mathrm{g} / \mathrm{ml})$ to IL-10. Results are shown as mean of triplicates \pm SE. 


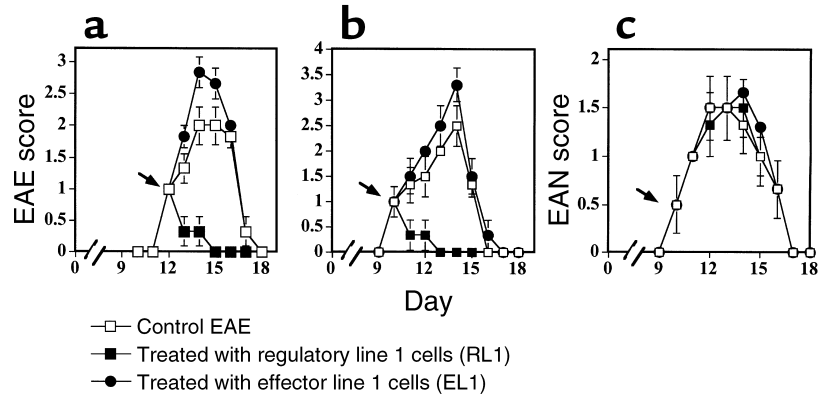

Figure 5

Groups of six Lewis rats were injected with MBPp87-99/CFA (a) and MBPp68-86/CFA (b) to induce active EAE, or with P2p57-81/CFA to induce active EAN (c). Just after the onset of each disease $(12,10$, and 10 days, respectively) these rats were administered (intravenously) $3 \times 10^{6} \mathrm{EL} 1$ or RL1 activated line cells. An observer blind to the experimental protocol monitored clinical manifestation of disease. Mean EAE score \pm SE of six rats per group is shown.

$\mathrm{pg} / \mathrm{ml}, 95 \pm 18 \mathrm{pg} / \mathrm{ml}, 26.5 \pm 7 \mathrm{ng} / \mathrm{ml}$, and $210 \pm 32$ $\mathrm{pg} / \mathrm{ml}$, respectively, in response to $100 \mu \mathrm{g} / \mathrm{ml}$ MBPp68-86). While W-2 expended easily during Agspecific selection cycles of the 11 high IL-10-producing clones only one clone (clone W-11) could survive selection cycles to allow an in vivo transfer experiment. This clone produced high levels of IL-10 and low levels of IL-4, IFN- $\gamma$, and TGF- $\beta$ (Table 1, 2,200 \pm 140 $\mathrm{pg} / \mathrm{ml}, 130 \pm 14 \mathrm{pg} / \mathrm{ml}, 8.2 \pm 0.4 \mathrm{ng} / \mathrm{ml}$, and $230 \pm 25$ $\mathrm{pg} / \mathrm{ml}$, respectively, in response to $100 \mu \mathrm{g} / \mathrm{ml}$ MBPp68-86). This clone resembled a $\operatorname{Tr} 1$ cell type, as has been characterized recently by Groux et al. (36). Rats with developing MBPp87-99/CFA-induced EAE, administered just after the onset of disease with $2 \times 10^{6}$ cells from clones $\mathrm{W}-11$, but not $\mathrm{W}-2$, went into fast remission (Figure 6, $P<0.001)$. Anti-IL-10- $(100 \mu \mathrm{g} / \mathrm{rat})$ but not anti-IL-4-neutralizing Ab's could totally abrogate this effect (Figure 6). Similar results were obtained in treating MBPp68-86/CFA-induced EAE (data not shown). Also, similar to RL1, these cells (at a dose of $2 \times 10^{6}$ per rat) had no effect on the manifestation of EAN (data not shown). This further demonstrates the role of $\operatorname{Tr} 1$ cells in the regulation of autoimmunity.

\section{Discussion}

In organ-specific autoimmune diseases such as MS, rheumatoid arthritis, and insulin-dependent diabetes mellitus, there are vigorous debates discussing what antigen triggers the autoimmune process and what antigens dominate the diverse immune response during determinant spread. It is rational, although it has yet to be proven, that antigen-specific regulatory cells are involved in the regulation of the autoimmune response mounted against autoimmune determinants, including those to which the response spreads. Eight years ago we demonstrated that administration of the soluble form of the encephalitogenic determinant rapidly reverses an ongoing disease induced by the same determinant (14). This process includes an intracellu- lar elevation of $\mathrm{Ca}^{++}$at the target autoimmune cells (15). Neutralizing Ab's to IL-4 could abrogate the tolerant state (16). This suggests that the tolerant state induced by soluble peptide therapy includes elicitation of regulatory $\mathrm{T}$ cells. The rapid effect of soluble peptide therapy may also suggest that this therapy boosts a preexisting regulatory response. Later on, Yu et al. demonstrated that administration of the soluble determinant to which the immune response spread could prevent relapsing episodes in SJL mice (17). In the current study we have used the well-defined model of EAE in the Lewis rat to demonstrate the role of high IL-10-producing T cells in the regulation of determinant spread. Four major types of $\mathrm{CD}^{+}$regulatory $\mathrm{T}$ cells have been of a major interest so far: Th2 cells that produce IL-4, and to a much lesser extent, TNF- $\alpha$ or IFN- $\gamma(19-29)$; high TGF- $\beta$-producing Th 3 cells that were associated with oral tolerance $(33,34) ; \operatorname{Tr} 1$ cells that were found to manifest a regulatory role in an experimental model of colitis (36); and $\mathrm{CD} 4^{+} \mathrm{CD} 25^{+}$ immunoregulatory $\mathrm{T}$ cells (37-40). The regulatory role of each of these cells in $\mathrm{T}$ cell-mediated autoimmunity has been the focus of numerous studies during the last few years (for a recent review see ref. 48). Of these, much has been studied about the role of the Th1/Th2 balance in the regulation of $\mathrm{T}$ cell-mediated autoimmunity. Alteration of the Th1/Th2 balance into Th2 were found capable of suppressing numerous $T$ cell-mediated autoimmune disease such as EAE type I diabetes or experimentally induced arthritis. Hence, it is not fully understood whether alteration of the Th1/Th2 balance toward Th2 is beneficial due to the fact that these cells are regulatory cells, because their selection competes with Th1 selection, or because cytokines produced by these cells are essential for the function of other regulatory $T$ cells. Our current study clearly shows that anti-IL-4 Ab could reverse (in vivo) the tolerant state induced by the determinant with which disease was induced (Figure 1). Because Ag-specific Th 2 cells are major producers of IL-4, it is plausible that they play a major role in tolerance induced by

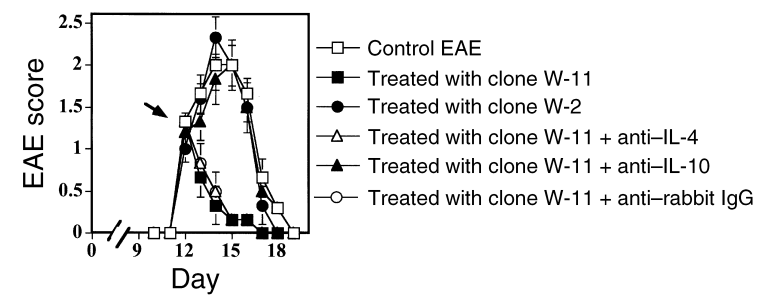

\section{Figure 6}

Groups of six Lewis rats were injected with MBPp87-99/CFA to induce active EAE. Just after the onset of each disease (12 days) these rats were administered (intravenously) $2 \times 10^{6} \mathrm{~W}-11$ or W-2 CD4 ${ }^{+} \mathrm{T}$ cell clones. To ensure the purity of clones $\mathrm{W}-11$ and $\mathrm{W}-2$ that were obtained by limited dilution each clone was subjected to RT-PCR with consensus primers (44), followed by sequencing of the VDJ region. An observer blind to the experimental protocol monitored clinical manifestation of disease. Mean EAE score \pm SE of six rats per group is shown. 
therapy with the epitope with which disease is established (Figure 1). We show here that neutralizing Ab to IL-4 could not reverse the suppressed response of T cells isolated from these protected animals in an ex vivo experiment. This suggests that in contrast to IL-10 or TGF- $\beta$, IL- 4 is not a suppressor cytokine. It is thus conceivable that Th2 (or Th2-like) IL-4-producing $\mathrm{T}$ cells induced by soluble peptide therapy are required for the maintenance of other, yet to be identified, regulatory suppressor cells. We do not exclude the possibility that $\mathrm{CD} 4^{+} \mathrm{CD} 25^{+}$immunoregulatory $\mathrm{T}$ cells participate in this complex regulatory system.

Our study brings about compelling evidence demonstrating the role of $\operatorname{Tr} 1$ cells in the expansion of tolerance to the spread determinant. At first, an apparent response to the major encephalitogenic determinant (p68-86) could be recorded in rats immunized with p87-99/CFA to develop active EAE (Table 1). We have also shown that as disease progresses this response is significantly higher than the one against the determinant with which disease was induced (Table 1). A T cell line (line EL1) that was selected in response to p68-87 from rats that were immunized only with p87-99/CFA was found to be encephalitogenic and mono specific (i.e., proliferates in response to $\mathrm{p} 68-86$ and not $\mathrm{p} 87-99$ ) even at early stages of selection. This clearly shows that even the acute model of MS in the Lewis rat includes determinant spread of pathogenic $\mathrm{T}$ cells. This further emphasizes the importance of a regulatory mechanism that restrains the activity of these cells. The current study clearly shows that neutralizing Ab to IL-10 can reverse tolerance induced by the spread determinant. $\operatorname{Tr} 1$ cells could be identified in cervical LNCs and spleen of EAE rats (Figure 3). Their number rapidly accelerated following soluble peptide therapy with the spread determinant (Figure 3 ). This may suggest that soluble peptide therapy accelerates a preexisting regulatory response and can provide a partial explanation for the very fast remission that follows soluble peptide therapy (Figure 1).

The therapeutic potential of IL-10 in EAE has been explored by numerous studies. IL-10 antagonizes the disease-promoting effects of IL-12 (49). PLP-specific T cells transfected to overexpress IL-10 could treat EAE in a targeted manner (50). Keyhole limpet hemocyanin-specific IL-10-producing Th2 cells could achieve this as well (51). IL-10-deficient mice are more susceptible and develop a more severe EAE when compared with IL-4-deficient mice or wild-type mice (52), and IL-10 transgenic mice are less susceptible to EAE than control mice (53). Anti-IL-10 Ab's reversed the inhibitory effect of intranasal administration of an MBP-derived peptide (54), and macrophage-produced IL-10 increased the ability of Ig chimera carrying the encephalitogenic determinant of PLP to treat ongoing EAE (55). Another regulatory cytokine of interest is TGF- $\beta$. Similar to IL-10, TGF- $\beta$ is also a regulatory cytokine, which suppresses the function of effector $T$ cells $(33,34)$. This cytokine is produced by regulatory
T cells and probably plays a part in the natural regulation of T cell-mediated autoimmunity (56-58). Furthermore, Groux et al. clearly demonstrated that the combination of anti-IL-10 and anti-TGF- $\beta$ best abrogates suppression induced by high IL-10-producing regulatory $\mathrm{T}$ cells (36). The reason that the current study focuses on IL-10 is that anti-IL-10 Ab could totally abrogate the protective effect induced by the soluble peptide therapy when used in conjunction with a determinant to which the immune response spreads (Figure 1).

Our results demonstrate the role of IL-10- and IL10 -producing $\mathrm{T}$ cells activated in response to the determinant to which the immune response spreads in the regulation of $\mathrm{T}$ cell tolerance. Their in vivo appearance during the course of disease, and their rapid induction following soluble peptide therapy (Figure 3) to provide protective immunity within 24 hours (Figure 1), may suggest that soluble peptide therapy amplifies a preexisting response in which, during in vivo activation, part of the $\mathrm{T}$ cells become regulatory cells. The finding that tolerance cannot be induced in response to an encephalitogenic determinant to which the immune response does not spread (PLPp224-240) further supports this hypothesis. It remains still to be elucidated whether IL-10-dependent suppression (Figure 3) and disease resistance (Figure 1) result from a direct effect of IL-10 produced by these cells on other effector $\mathrm{T}$ cells or via other regulatory $\mathrm{T}$ cells that exert an IL-10-dependent function.

It is interesting that tolerance with the inducing peptide leads to IL-4 production, while tolerance to the spread epitope leads to IL-10 production. Consequently, anti-IL-4 Ab abrogates tolerance induced by the determinant with which disease was established, whereas anti-IL-10 reverses tolerance induced by the spread epitope. The mechanistic basis of how therapy with one epitope boosts IL-4-producing T cells and with the other epitope eliciting IL-10-producing regulatory cells is not fully understood and will be in the focus of our future studies.

The protective effect of our regulatory line, RL1, on both p87-99- and p68-86-induced EAE, but not on EAN, indicates a disease-specific effect. Figure 4 shows that these regulatory cells, once activated, can suppress immune responses in a bystander manner and no longer retain determinant-specific inhibition (Figure 4a). Yet the presence of their target determinant further amplifies their suppressive capacity (Figure 4a). It is thus plausible that the in vivo specificity of their regulatory function could be attributed to the presence of their target determinant at the autoimmune site, which directs their selective propagation and accumulation, and at the site of inflammation. At this site they can therefore effectively suppress the function of autoimmune $T$ cells by soluble factors and/or cell-cell interaction. The contribution of cell-cell interaction to the suppressive function of regulatory cells has been demonstrated previously for $\mathrm{CD} 4^{+} \mathrm{CD} 25^{+} \mathrm{T}$ regulatory 
cells (37) and very recently for $\mathrm{CD} 8^{+} \mathrm{CD} 28^{-} \mathrm{T}$ regulatory cells (59). It will also be a focus of our future studies aimed to further explore the mechanistic basis of tolerance induced by soluble peptide therapy, and how it interferes in the complex interactions between various regulatory $\mathrm{T}$ cells $(60)$.

\section{Acknowledgments}

This study was supported by The Israel Cancer Research Fund, Israel Science Foundation, the Fund for Research Promotion of the Technion, and the Vice President Research Hedson Fund.

1. Wekerle, H. 1993. Experimental autoimmune encephalomyelitis as a model of immune-mediated CNS disease. Curr. Opin. Neurobiol. 3:779-784.

2. Raine, C.S. 1991. Multiple sclerosis: a pivotal role for the T cell in lesion development. Neuropathol. Appl. Neurobiol. 17:265-274.

3. McRae, B.L., Vanderlugt, C.L., Dal Canto, M.C., and Miller, S.D. 1995. Functional evidence for epitope spreading in the relapsing pathology of experimental autoimmune encephalomyelitis. J. Exp. Med. 182:75-85.

4. Offner, H., et al. 1989. T cell determinants of myelin basic protein include a unique encephalitogenic I-E-restricted epitope for Lewis rats. J. Exp. Med. 170:355-367.

5. Offner, H., et al. 1992. Characterization of the immune response to a secondary encephalitogenic epitope of basic protein in Lewis rats. I. $\mathrm{T}$ cell receptor peptide regulation of $\mathrm{T}$ cell clones expressing cross-reactive $\mathrm{V}$ beta genes. J. Immunol. 148:1706-1711.

6. Lehmann, P.V., Forsthuber, T., Miller, A., and Sercarz, E.E. 1992. Spreading of T-cell autoimmunity to cryptic determinants of an autoantigen. Nature. 358:155-157.

7. Mor, F., and Cohen, I.R. 1993.Shifts in the epitopes of myelin basic protein recognized by Lewis rat $\mathrm{T}$ cells before, during, and after the induction of experimental autoimmune encephalomyelitis. J. Clin. Invest. 92:2199-2206.

8. Miller, S.D., et al. 1995. Blockade of CD28/B7-1 interaction prevents epitope spreading and clinical relapses of murine EAE. Immunity. 3:739-745.

9. Vandenbark, A.A., et al. 1994. Definition of encephalitogenic and immunodominant epitopes of Guinea pig myelin basic protein (Gp$\mathrm{BP})$ in Lewis rats tolerized neonatally with Gp-BP or Gp-BP peptides. J. Immunol. 153:852-861.

10. Clayton, J.P., et al. 1989. Peptide-specific prevention of experimental allergic encephalomyelitis. Neonatal tolerance induced to the dominant T cell determinant of myelin basic protein. J. Exp. Med. 169:1681-1691.

11. Grabie, N., Wohl, I., Youssef, S., Wildbaum, G., and Karin, N. 1999 Expansion of neonatal tolerance to self in adult life: I. The role of a bacterial adjuvant in tolerance spread. Int. Immunol. 11:899-906.

12. Grabie, N., and Karin, N. 1999. Expansion of neonatal tolerance to self in adult life: II. Tolerance preferentially spreads in an intramolecular manner. Int. Immunol. 11:907-913.

13. Zhao, W., Wegmann, K.W., Trotter, J.L., Ueno, K., and Hickey, W.F. 1994. Identification of an $\mathrm{N}$-terminally acetylated encephalitogenic epitope in myelin proteolipid apoprotein for the Lewis rat. J. Immunol. 153:901-909.

14. Karin, N., Mitchell, J.D., Brocke, S., Ling, N., and Steinman, L. 1994 Reversal of experimental autoimmune encephalomyelitis by as soluble peptide variant of a myelin basic protein epitope: $T$ cell receptor antagonism and reduction of IFN-g and TNF-a production. J. Exp. Med. 180:2227-2237.

15. Karin, N., et al. 1998. Short peptide based tolerogens without antigenic activity reverse autoimmunity. J. Immunol. 160:5188-5149.

16. Brocke, S., et al. 1996. Treatment of experimental encephalomyelitis with a peptide analogue of myelin basic protein. Nature. 379:343-346.

17. Yu, M., Johnson, J.M., and Tuohy, V.K. 1996. A predictable sequential determinant spreading cascade invariably accompanies progression of experimental autoimmune encephalomyelitis: a basis for peptide-specific therapy after onset of clinical disease. J. Exp. Med. 183:1777-1788.

18. Vanderlugt, C.L., et al. 2000. Pathologic role and temporal appearance of newly emerging autoepitopes in relapsing experimental autoimmune encephalomyelitis. J. Immunol. 164:670-678.

19. O'Garra, A., and Murphy, K. 1994. Role of cytokines in determining T-lymphocyte function. Curr. Opin. Immunol. 6:458-466.

20. Abbas, A.K., Murphy, K.M., and Sher, A. 1996. Functional diversity of helper T lymphocytes. Nature. 383:787-793.
21. Swain, S.L., Weinberg, A.D., English, M., and Huston, G. 1990. IL-4 directs the development of Th2-like helper effectors. J. Immunol. 145:3796-3806.

22. Lederer, J.A., et al. 1996. Cytokine transcriptional events during helper T cell subset differentiation. J. Exp. Med. 184:397-406.

23. Seder, R.A., Gazzinelli, R., Sher, A., and Paul,W.E. 1993. IL-12 acts directly on CD4+ T cells to enhance priming for IFN- $\gamma$ production and diminishes IL-4 inhibition of such priming. Proc. Natl. Acad. Sci. USA. 90:10188-10192.

24. Seder, R.A., Paul, W.E., Davis, M.M., and Fazekas de St. Groth, B. 1992. The presence of interleukin-4 during in vitro priming determines the cytokine-producing potential of CD4+ T cells from $\mathrm{T}$ cell receptor transgenic mice. J. Exp. Med. 176:1091-1098.

25. Fiorentino, D.F., et al. 1991. IL-10 acts on the antigen presenting cell to inhibit cytokine production by Th1 cells. J. Immunol. 146:3444-3451.

26. Mosmann, T.R., and Coffman, R.L. 1989. Th1 and Th2 cells: different patterns of lymphokine secretion lead to different functional properties. Annu. Rev. Immunol. 9:145-173.

27. Mosmann, T., and Moor, K. 1989. The role of IL-10 in the crossregulation of Th1 and Th2 responses. Immunol. Today. 12:A49-A53.

28. Huang, H., Hu-Li, J., Chen, H., Ben-Sasson, S.Z., and Paul, W.E. 1997. IL-4 and IL-13 production in differentiated T helper type 2 cells is not IL-4 dependent. J. Immunol. 159:3731-3738.

29. Robinson, D., et al. 1997. IGIF does not drive Th1 development but synergizes with IL-12 for interferon-gamma production and activates IRAK and NFkappaB. Immunity. 7:571-581.

30. Leonard, J.P., Waldburger, K.E., and Goldman, S.J. 1995. Prevention of experimental autoimmune encephalomyelitis by antibodies against interleukin 12. J. Exp. Med. 181:381-386.

31. Wildbaum, G., Youssef, S., Grabie, N., and Karin, N. 1998. Prevention of experimental autoimmune encephalomyelitis by antibodies to interferon gamma inducing factor. J. Immunol. 161:6368-6374.

32. Wildbaum, G., Netzer, N., and Karin, N. 2002. Plasmid DNA encoding IFN-gamma-inducible protein 10 redirects antigen-specific $T$ cell polarization and suppresses experimental autoimmune encephalomyelitis. J. Immunol. 168:5885-5892.

33. Chen, Y., Kuchroo, V.K., Inobe, J., Hafler, D., and Weiner, H.L. 1994. Regulatory T-cell clones induced by oral tolerance: Suppression of autoimmune encephalomyelitis. Science. 265:1237-1240.

34. Seddon, B., and Mason, D. 1999. Regulatory T cells in the control of autoimmunity: the essential role of transforming growth factor beta and interleukin 4 in the prevention of autoimmune thyroiditis in rats by peripheral CD4(+)CD45RC- cells and CD4(+)CD8(-) thymocytes. J. Exp. Med.. 189:279-288.

35. Nicholson, L.B., Greer, J.M., Sobel, R.A., Lees, M.B., and Kuchroo, V.K. 1995. An altered peptide ligand mediates immune deviation and prevents autoimmune encephalomyelitis. Immunity. 3:397-405.

36. Groux, H., et al. 1997. A CD4+ T-cell subset inhibits antigen-specific T-cell responses and prevents colitis. Nature. 389:737-742.

37. Thornton, A.M., and Shevach, E.M. 1998.CD4+CD25+ immunoregulatory $\mathrm{T}$ cells suppress polyclonal $\mathrm{T}$ cell activation in vitro by inhibiting interleukin 2 production. J. Exp. Med. 188:287-296.

38. Salomon, B., et al. 2000. B7/CD28 costimulation is essential for the homeostasis of the CD4+CD25+ immunoregulatory T cells that control autoimmune diabetes. Immunity. 12:431-440.

39. Shevach, E.M. 2002. CD4+ Cd25+ suppressor T cells: more questions than answers. Nat. Rev. Immunol. 2:389-400.

40. Takahashi, T., et al. 2000. Immunologic self-tolerance maintained by $\mathrm{CD} 25(+) \mathrm{CD} 4(+)$ regulatory $\mathrm{T}$ cells constitutively expressing cytotoxic T lymphocyte-associated antigen 4. J. Exp. Med. 192:303-310.

41. Olee, T., Powers, J.M., and Brostoff, S.W. 1988. A T cell epitope for experimental allergic neuritis. J. Neuroimmunol. 19:167-173.

42. Youssef, S., et al. 1998. Long lasting protective immunity to experimental autoimmune encephalomyelitis following vaccination with naked DNA encoding C-C chemokines. J. Immunol. 161:3870-3879.

43. Ben-Nun, A., Wekerle, H., and Cohen, I.R. 1981. The rapid isolation of clonable antigen-specific $\mathrm{T}$ lymphocyte lines capable of mediating autoimmune encephalomyelitis. Eur. J. Immunol. 11:195-199.

44. Karin, N., Szafer, F., Mitchell, D., Gold, D.P., and Steinman, L. 1993. Selective and nonselective stages in homing of T lymphocytes to the central nervous system during experimental allergic encephalomyelitis. J. Immunol. 150:4116-4124.

45. Wildbaum, G., and Karin, N. 1999. Augmentation of natural immunity to a pro-inflammatory cytokine (TNF-alpha) by targeted DNA vaccine confers long-lasting resistance to experimental autoimmune encephalomyelitis. Gene Ther. 6:1128-1138.

46. Wildbaum, G., Westermann, J., Maor, G., and Karin, N. 2000. A targeted DNA vaccine encoding Fas ligand defines its dual role in the regulation of experimental autoimmune encephalomyelitis. J. Clin. Invest. 106:671-679. 
47. Openshaw, P., et al. 1995. Heterogeneity of intracellular cytokine synthesis at the single-cell level in polarized T helper 1 and $T$ helper 2 populations. J. Exp. Med. 182:1357-1367.

48. Maloy, K.J., and Powrie, F. 2001. Regulatory T cells in the control of immune pathology. Nat. Immunol. 2:816-822.

49. Segal, B.M., Dwyer, B.K., and Shevach, E.M. 1998. An interleukin (IL)-10/IL-12 immunoregulatory circuit controls susceptibility to autoimmune disease. J. Exp. Med. 187:537-546.

50. Mathisen, P.M., Yu, M., Johnson, J.M., Drazba, J.A., and Tuohy, V.K. 1997. Treatment of experimental autoimmune encephalomyelitis with genetically modified memory T cells. J. Exp. Med. 186:159-164.

51. Stohlman, S.A., Pei, L., Cua, D.J., Li, Z., and Hinton, D.R. 1999. Activation of regulatory cells suppresses experimental allergic encephalomyelitis via secretion of IL-10. J. Immunol. 163:6338-6344.

52. Bettelli, E., et al. 1998. IL-10 is critical in the regulation of autoimmune encephalomyelitis as demonstrated by studies of IL-10- and IL-4-deficient and transgenic mice. J. Immunol. 161:3299-3306.

53. Cua, D.J., Groux, H., Hinton, D.R., Stohlman, S.A., and Coffman, R.L. 1999. Transgenic interleukin 10 prevents induction of experimenta autoimmune encephalomyelitis. J. Exp. Med. 189:1005-1010.

54. Burkhart, C., Liu, G.Y., Anderton, S.M., Metzler, B., and Wraith, D.C.
1999. Peptide-induced T cell regulation of experimental autoimmune encephalomyelitis: a role for IL-10. Int. Immunol. 11:1625-1634.

55. Legge, K.L., et al. 2000. Coupling of peripheral tolerance to endogenous interleukin 10 promotes effective modulation of myelin-activated $\mathrm{T}$ cells and ameliorates experimental allergic encephalomyelitis. J. Exp. Med. 191:2039-2052.

56. Bridoux, F., et al. 1997. Transforming growth factor beta (TGF-beta)dependent inhibition of T helper cell 2 (Th2)-induced autoimmunity by self-major histocompatibility complex (MHC) class II-specific, regulatory CD4(+) T cell lines. J. Exp. Med. 185:1769-1775.

57. Adlard, K., et al. 1999. Immunoregulation of encephalitogenic MBPNAc1-11-reactive T cells by CD4+ TCR-specific T cells involves IL-4 IL-10 and IFN-gamma. Autoimmunity. 31:237-248.

58. Bebo, B.F., Jr., et al. 1999. Gender differences in protection from EAE induced by oral tolerance with a peptide analogue of MBP-Ac1-11. J. Neurosci. Res. 55:432-440.

59. Chang, C.C., et al. 2002. Tolerization of dendritic cells by T(S) cells: the crucial role of inhibitory receptors ILT3 and ILT4. Nat. Immunol. 3:237-243.

60. Sakaguchi, S. 2000. Regulatory T cells: key controllers of immunologic self-tolerance. Cell. 101:455-458. 